Supplement of Mech. Sci., 7, 247-253, 2016

http://www.mech-sci.net/7/247/2016/

doi:10.5194/ms-7-247-2016-supplement

(C) Author(s) 2016. CC Attribution 3.0 License.

(c) (i)

Supplement of

\title{
A structure design method for compliant parallel manipulators with actuation isolation
}

Guangbo Hao and Xianwen Kong

Correspondence to: Guangbo Hao (g.hao@ucc.ie)

The copyright of individual parts of the supplement might differ from the CC-BY 3.0 licence. 


\section{Supplemental material: A 6-DOF CPM case with actuation isolation and its derived lower-DOF CPM}

A 6-DOF CPM with actuation isolation (Fig. S.1(a)) can be proposed using the proposed actuation-leg addition method where the original compliant mechanism is a 0-DOC (6-DOF) mechanism (free rigid body). It is composed of six legs, each of which consists of a 1-DOF translational/prismatic joint and a 5-DOF wire beam with its axis along with the direction of the translational joint. For practical manufacturing reasons, the six wire beams arranged in three pairs along three orthogonal directionsse

In an $n$-DOF rigid-link parallel mechanism, we can obtain its derived mechanisms with DOF less than $n$ by imposing certain constraints, such as locking joints [S.1], on the mechanisms. By locking one or more translational joints or replacing one or more pair(s) of translational joints along the same direction with one translationaljointalong thesamedirectiontoconnecttotheassociatedpairof wirebeams, we canobtainanumber of derived 1 to 5 DOF CPMs with actuation isolation as elaborated in Figs. S.1(b)-S.1(q). As pointed out in [S.2], the use of 2T1R or alike may not represent the motion rigorously in some cases. The notation is still used in this paper considering it is still in common use in CPMs.

For example, the 3-DOF 3T CPM with actuation isolation shown in Fig. S.1(k) can be obtained from the 6-DOF CPM in Fig. S.1(a) by replacing each pair of two translational joints along the same direction by one translational joint connected to the associated pair of parallel wire beams. Figure S.2(a) shows a more detailed design of this CPM, where each actuated $\mathrm{T}$ joint uses the proposed joint in [S.3]. The FEA motion demonstrations for the translations along the X-, Y-, and Z-axes are shown in Figs. S.2(b), S.2(c), and S.2(d), respectively. It can be seen that one actuation along one axis does not produce transverse motions of another two linear actuators due to the guiding of translational joints and the decoupling from wire beams.

\section{References}

[S.1] Kong, X. and Yu, J., Type synthesis of 2-DOF 3-4R parallel mechanisms with both spherical translation mode and sphere-on-sphere rolling mode, ASME Journal of Mechanisms and Robotics, 2015, 7(4), 041018.

[S.2] Kong, X., and Gosselin, M., Type Synthesis of Parallel Mechanisms, Springer-Verlag, 2007

[S.3] Trease, B.P., Moon, Y., Kota, S., 2004, Design of Large-Displacement Compliant Joints. ASME Journal of Mechanical Design, 127(4):788-798. doi:10.1115/1.1900149. 


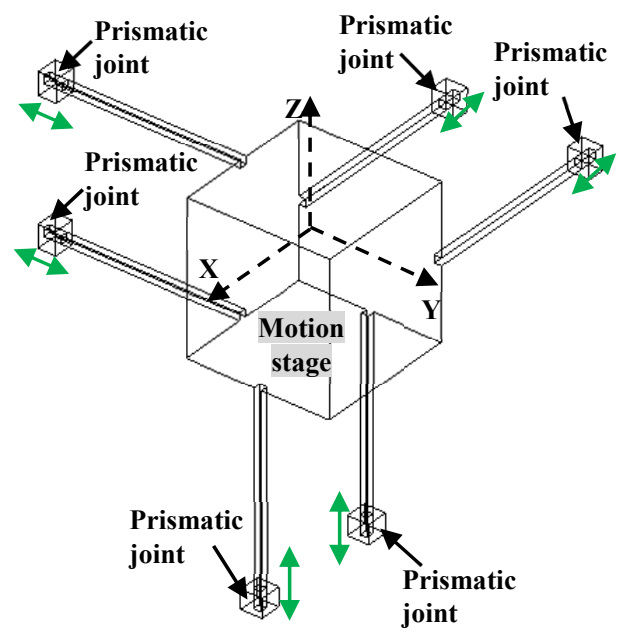

a) 6-DOF CPM

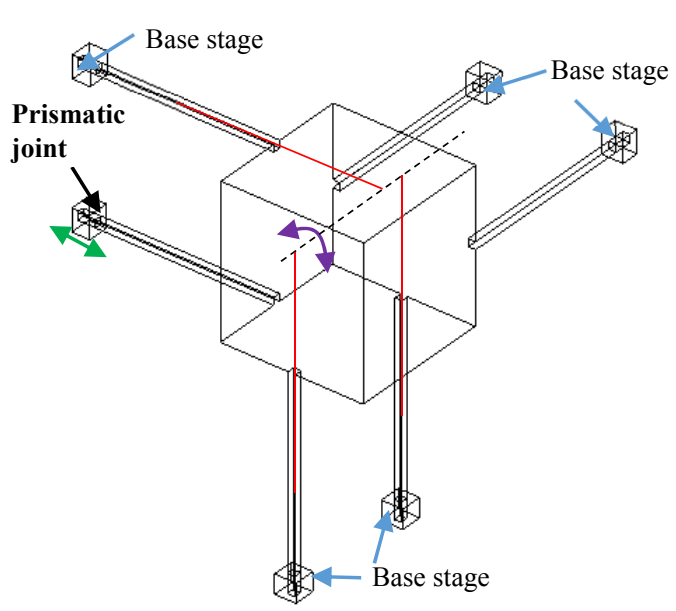

c) 1-DOF 1R CPM

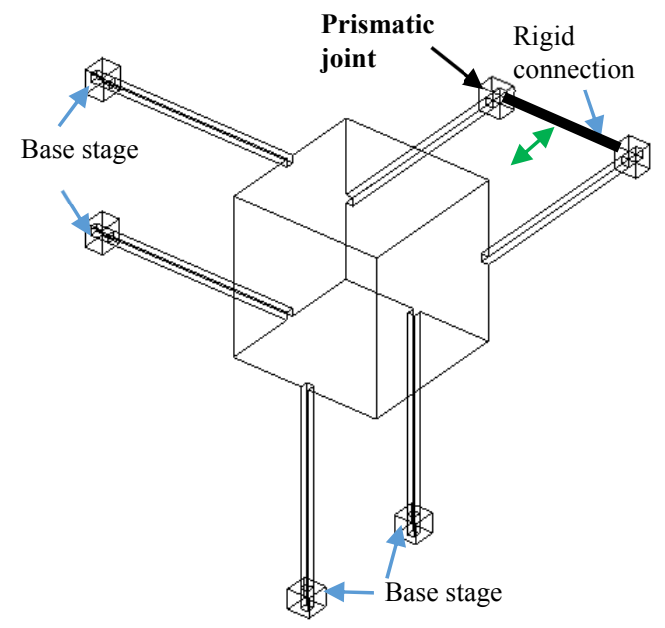

e) 1-DOF $1 \mathrm{~T} \mathrm{CPM}$

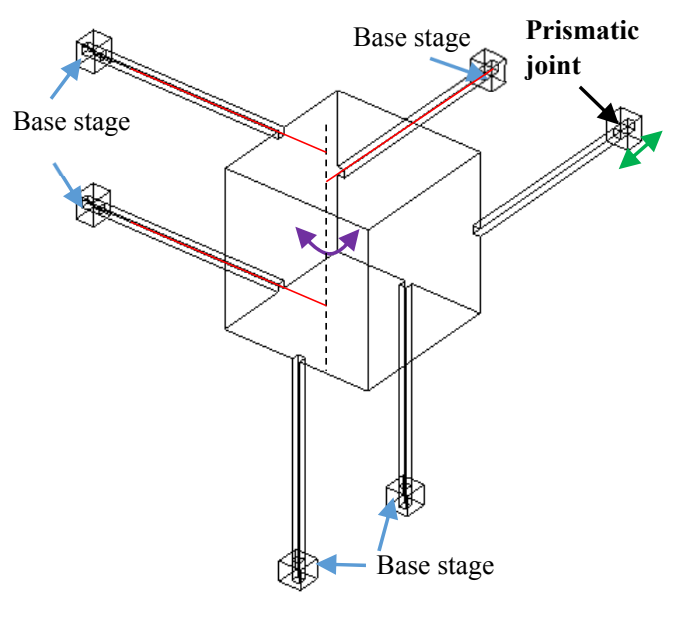

b) 1-DOF 1R CPM

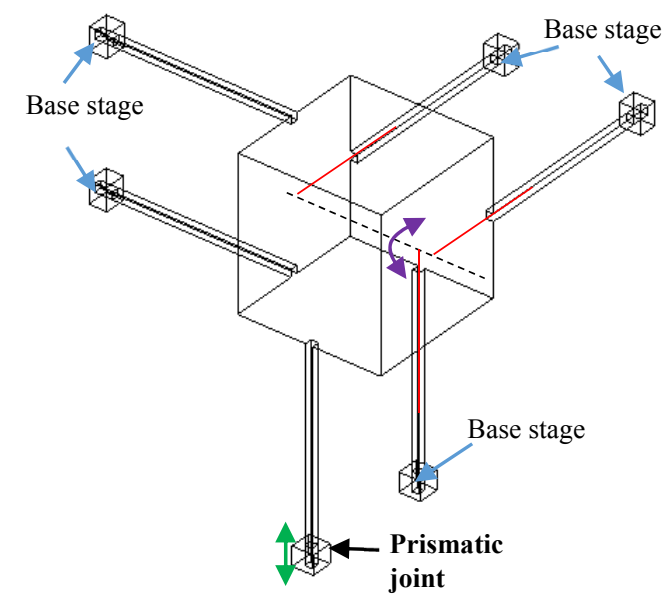

d) 1-DOF 1R CPM

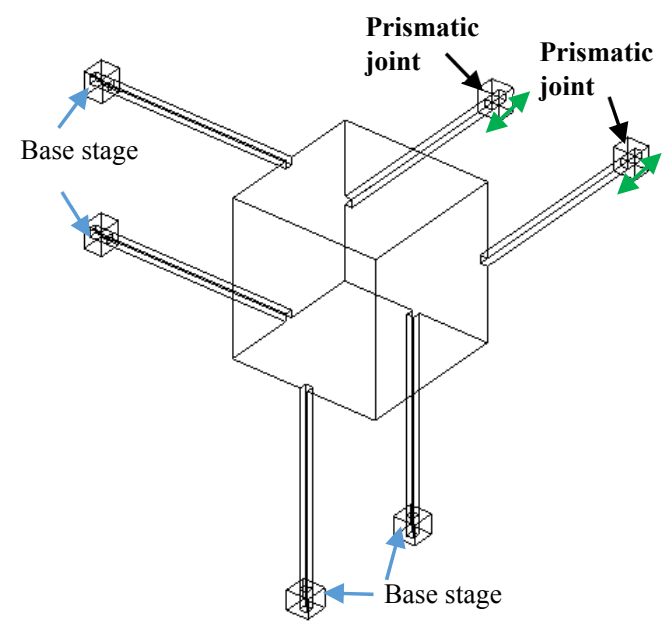

f) 2-DOF $1 \mathrm{~T} 1 \mathrm{R} \mathrm{CPM}$

Figure S.1: A 6-DOF CPMs with actuation isolation and its derived CPMs (Continued) 


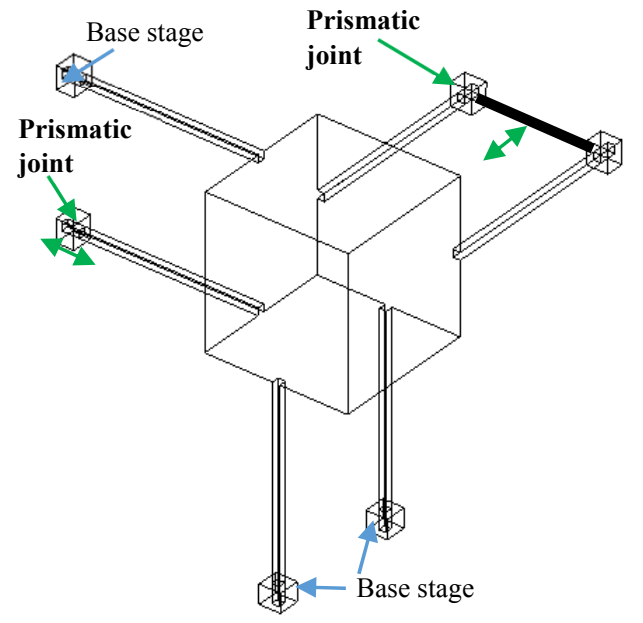

g) 2-DOF 1T1R CPM

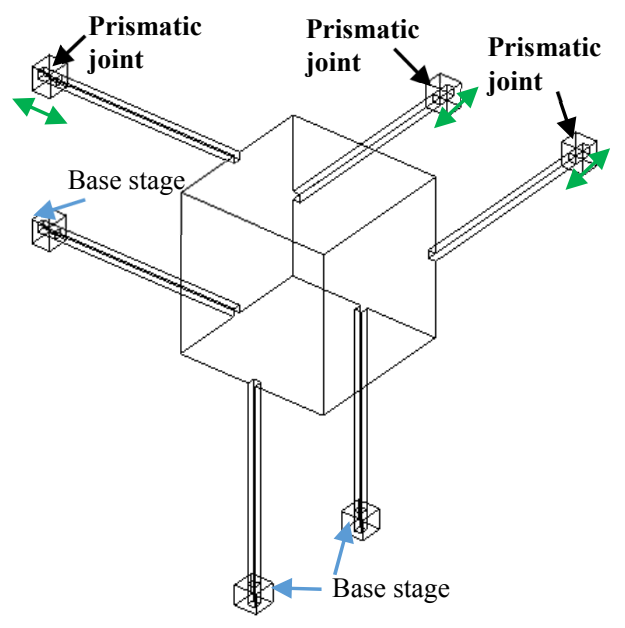

i) 3-DOF 2R1T CPM

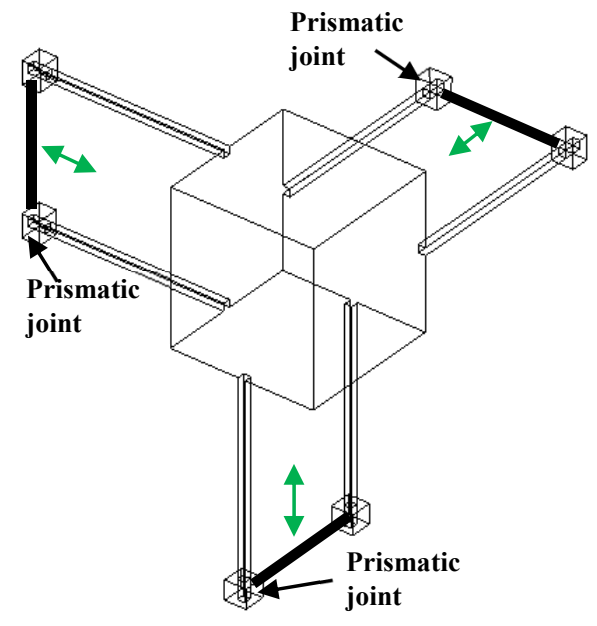

k) 3-DOF 3T CPM

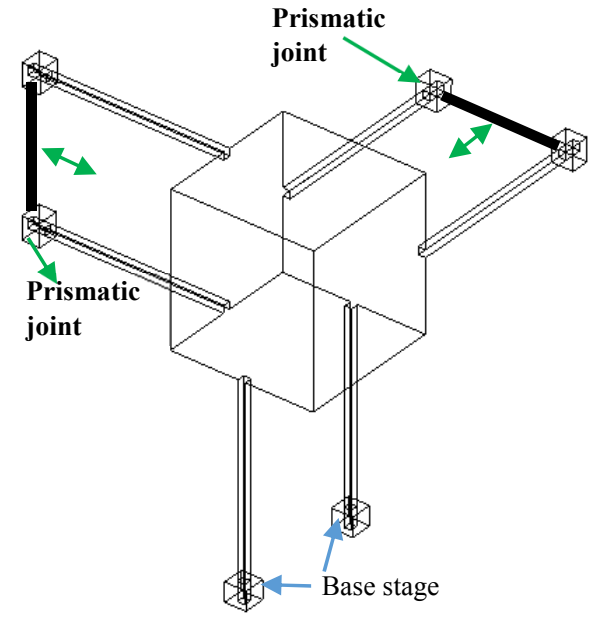

h) 2-DOF 2T CPM

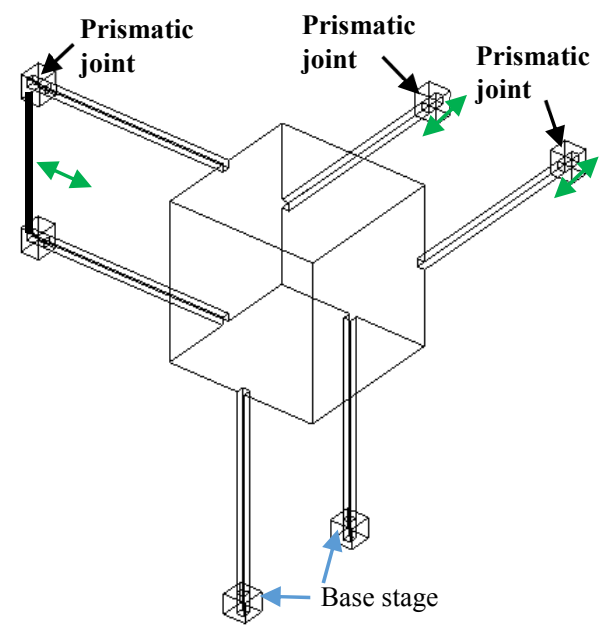

j) 3-DOF 2T1R CPM

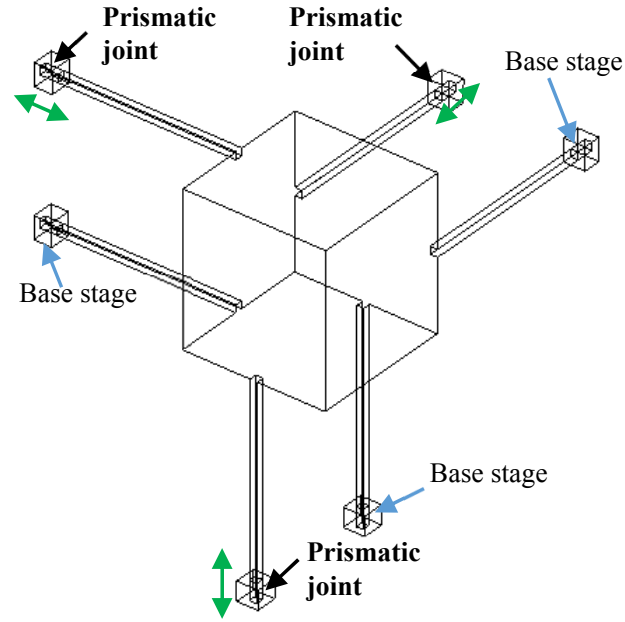

1) 3-DOF $3 R$ CPM

Figure S.1: A 6-DOF CPMs with actuation isolation and its derived CPMs (Continued) 


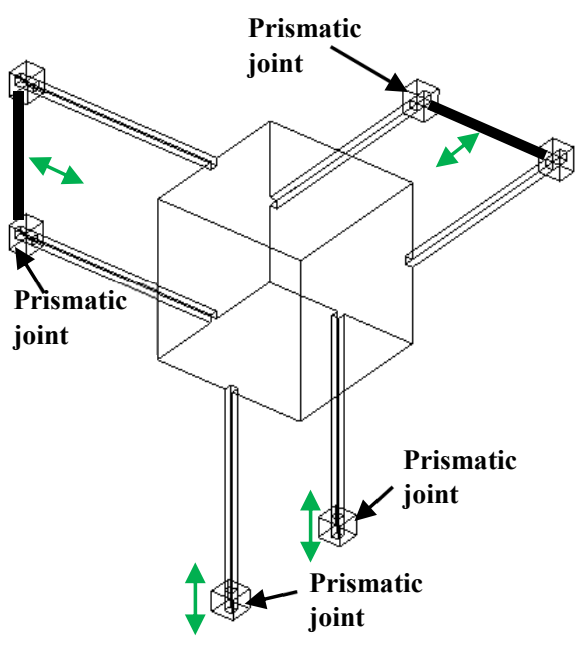

m) 4-DOF 3T1R CPM

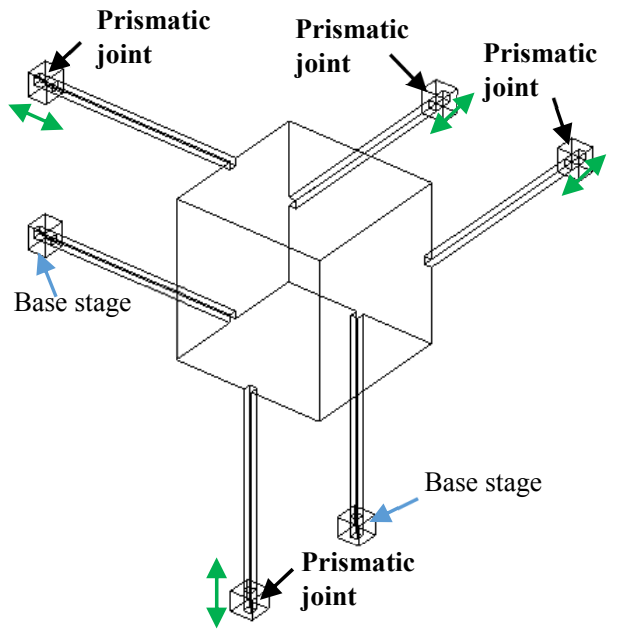

n) 4-DOF 3R1T CPM

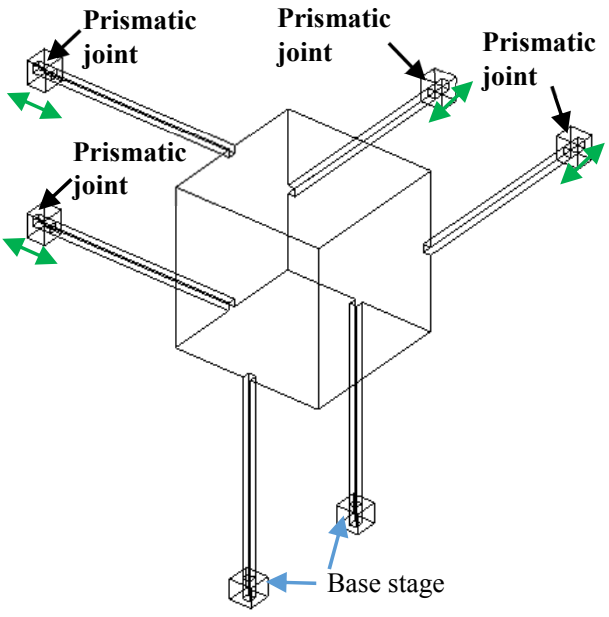

o) 4-DOF 2R2T CPM

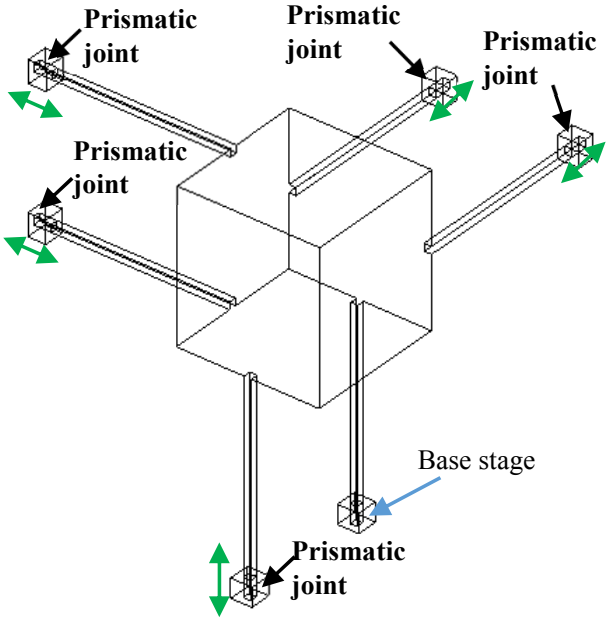

p) 5-DOF $3 \mathrm{R} 2 \mathrm{~T} \mathrm{CPM}$

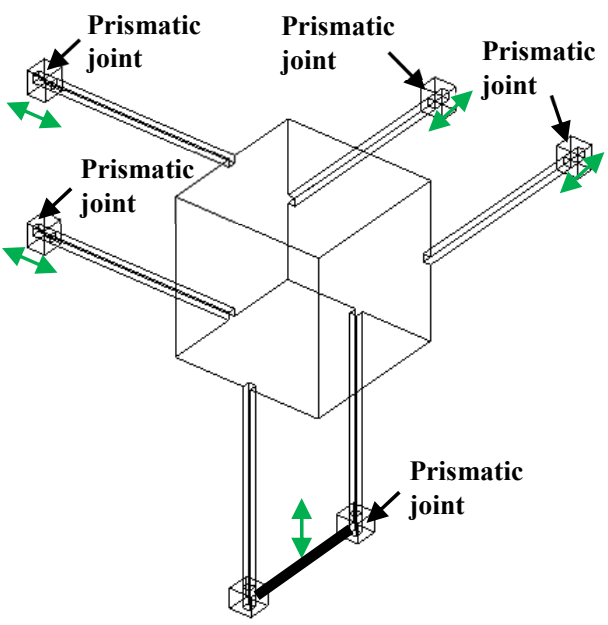

q) $5-\mathrm{DOF} 2 \mathrm{R} 3 \mathrm{~T} \mathrm{CPM}$

Figure S.1: A 6-DOF CPMs with actuation isolation and its derived CPMs 


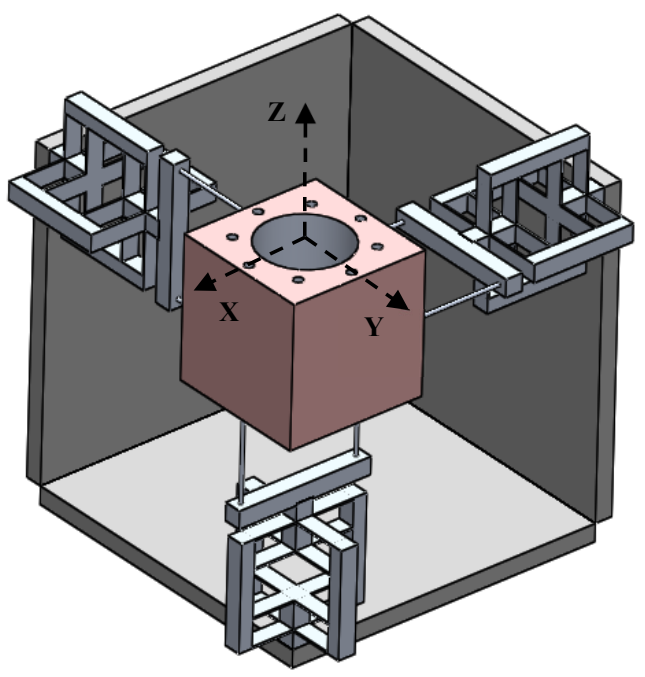

(a) Design of 3-DOF 3T CPM

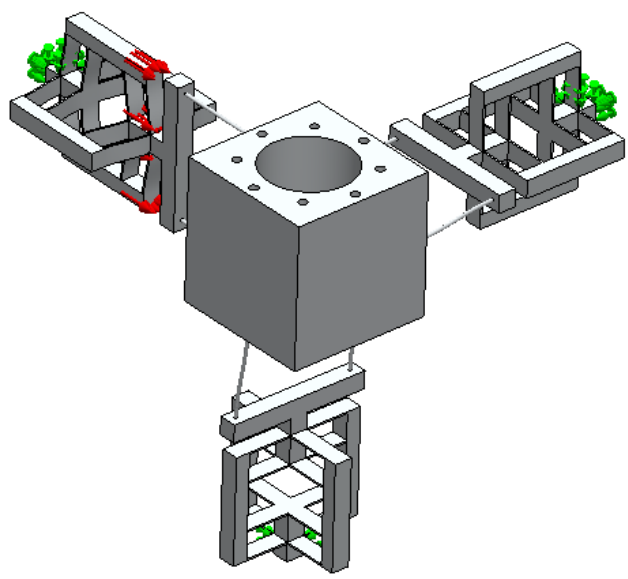

(c) FEA translational result by $\mathrm{Y}$-axis actuation

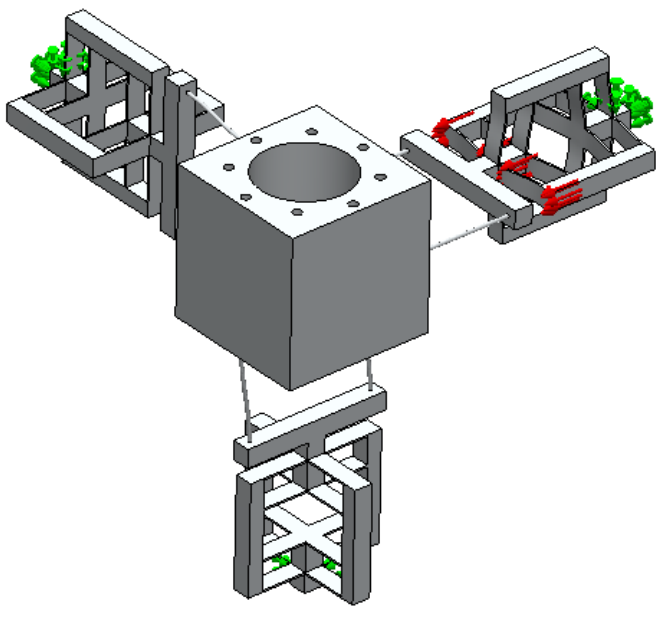

(b) FEA translational result by $\mathrm{X}$-axis actuation

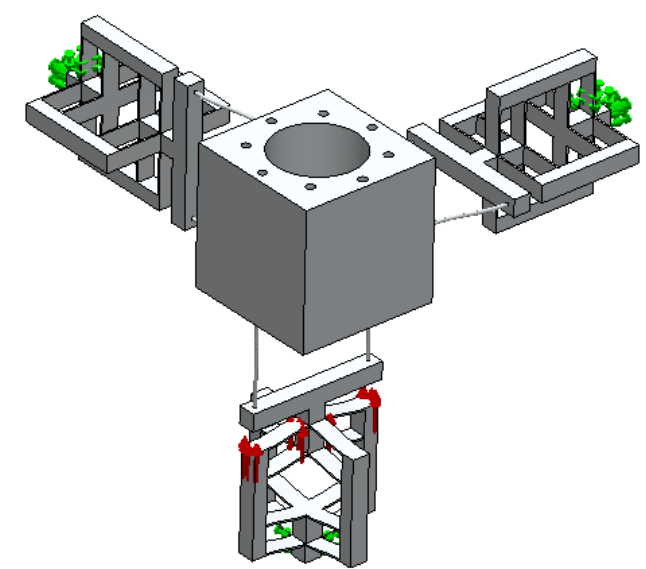

(d) FEA translational result by Z-axis actuation

Figure S.2: Derived 3-DOF 3T CPM with actuation isolation (Fig. S.1(k)) 